\section{AORTIC FILTRATION SYSTEM'S IMPACT ON PREVENTING ADVERSE CLINICAL EVENTS}

To the Editor:

In their recent article in the Journal, Ye and Webb ${ }^{1}$ reported their first-in-human clinical experience with a new version of the EMBOL-X intra-aortic filter (Edwards Lifesciences Corporation, Irvine, Calif) to capture embolic material during transcatheter aortic valve replacement and cardiac surgery in which the filter is not bundled with a perfusion cannula. In 2003, the Annals of Thoracic Surgery published the results of a multicenter, randomized trial with 1289 patients evaluating the safety and efficacy of the old EMBOL-X aortic filtration system (subsequently purchased by Edwards) in which the perfusion cannula and filter were combined. ${ }^{2}$ That earlier trial was a US Food and Drug Administration (FDA) investigative device exemption trial that was presented successfully as a premarket approval to the FDA Circulatory Device Panel. Ye and $\mathrm{Webb}^{1}$ are to be congratulated for reconfirming the efficacy of the old filter system at successfully capturing mircroemboli; however, we take issue with conclusions reported in their current article that they attribute to our original 2003 publication.

Ye and Webb ${ }^{1}$ correctly state that our article reported "emboli in $96.8 \%$ of filters removed after the procedure" but are incorrect in stating that we reported "a $74 \%$ reduction of cerebral injury in high-risk patients." In fact, despite a high embolic capture rate, adverse event rates were similar between the groups, including no significant differences in stroke, myocardial infarction, death, or renal complications (Table 1 [Table 4 in our 2003 article $^{2}$ ]). The 1289 patients in this earlier trial were generally at low risk, and in attempt to correlate emboli capture with clinical events we performed a post hoc analysis in which we identified $19 \%$ of the patients in our study as at moderate or high risk and looked at event rates with and without the filter in that specific population. Even in the high-risk subgroup, there was no difference in cerebrovascular events and certainly not a " $74 \%$ reduction" (Table 2 [Table 4 in our 2003 article $^{2}$ ]). In the post hoc analysis involving the high-risk group, we did demonstrate a significant reduction in renal complications, from $24 \%$ to $14 \%$, but the article of Ye and $\mathrm{Webb}^{1}$ does not provide appropriate context to this statistic and also incorrectly implies this was in the overall study.

\footnotetext{
The Editor welcomes submissions for possible publication in the Letters to the Editor section that consist of commentary on an article published in the Journal or other relevant issues. Authors should: • Include no more than 500 words of text, three authors, and five references. $\bullet$ Type with double-spacing. $\bullet$ See http://jtcs.ctsnetjournals.org/ misc/ifora.shtml for detailed submission instructions. • Submit the letter electronically via jtcvs.editorialmanager.com. Letters commenting on an article published in the JTCVS will be considered if they are received within 6 weeks of the time the article was published. Authors of the article being commented on will be given an opportunity of offer a timely response ( 2 weeks) to the letter. Authors of letters will be notified that the letter has been received. Unpublished letters cannot be returned.
}

TABLE 1. Adverse events in the International Council of Emboli Management 2000 investigation

\begin{tabular}{lccc}
\hline \multicolumn{1}{c}{ Event } & Filter $(\mathbf{N}=\mathbf{6 4 5})$ & Control $(\mathbf{N}=\mathbf{6 4 4})$ & $\boldsymbol{P}$ value \\
\hline Death & $10(1.6 \%)$ & $11(1.7 \%)$ & .82 \\
Stroke & $17(2.6 \%)$ & $14(2.2 \%)$ & .59 \\
TIA & $1(0.2 \%)$ & $4(0.6 \%)$ & .22 \\
Stroke or TIA & $18(2.8 \%)$ & $18(2.8 \%)$ & $>.999$ \\
RI & & & \\
$\quad$ Without dialysis & $33(5.1 \%)$ & $43(6.7 \%)$ & .23 \\
$\quad$ With dialysis & $7(1.1 \%)$ & $9(1.4 \%)$ & .61 \\
$\quad$ All RI & $40(6.2 \%)$ & $52(8.1 \%)$ & .19 \\
MI & $21(3.3 \%)$ & $18(2.8 \%)$ & .63 \\
$\quad$ Q-wave MI & $46(7.1 \%)$ & $46(7.1 \%)$ & .99 \\
$\quad$ CK-MB elevation & $67(10.4 \%)$ & $64(9.9 \%)$ & .79 \\
$\quad$ All MI & $5(0.8 \%)$ & $5(0.8 \%)$ & $>.999$ \\
Gastrointestinal & $110(17.1 \%)$ & $122(18.9 \%)$ & $>.999$ \\
Composite events & & & \\
\hline All
\end{tabular}

All data represent numbers and percentages of patients. TIA, Transient ischemic attack; $R I$, renal insufficiency; $M I$, myocardial infarction; $C K-M B$, creatine kinase isoenzyme MB. Adapted from Banbury MK, Kouchoukos NT, Allen KB, Slaughter MS, Weissman NJ, Berry GJ, et al; ICEM 2000 Investigators. Emboli capture using the Embol-X intraaortic filter in cardiac surgery: a multicentered randomized trial of 1,289 patients. Ann Thorac Surg. 2003;76:508-15; discussion 515.

Intuitively, embolic capture should reduce clinical events; however, well-designed clinical trials including the pivotal FDA trial for this device, have failed to tie the surrogate end point of embolic capture to better outcomes. This device was approved by the FDA Circulatory Device Panel because it was safe and made sense, not because it demonstrated a reduction in adverse events. As noted in the conclusion of our original article, "further study of high-risk patients is

TABLE 2. Individual end point events for patients with severity score of at least 5 in the International Council of Emboli Management 2000 investigation

\begin{tabular}{lccc}
\hline \multicolumn{1}{c}{ Event } & Filter $(\mathbf{N}=\mathbf{1 2 4})$ & Control $(\mathbf{N}=\mathbf{1 1 7})$ & $\boldsymbol{P}$ value \\
\hline Death & $4(3.2 \%)$ & $5(4.3 \%)$ & .67 \\
Stroke & $8(6.5 \%)$ & $6(5.1 \%)$ & .66 \\
TIA & $0(0.0 \%)$ & $0(0.0 \%)$ & - \\
RI & & & \\
$\quad$ Without dialysis & $13(10.5 \%)$ & $22(18.8 \%)$ & .07 \\
$\quad$ With dialysis & $4(3.2 \%)$ & $6(5.1 \%)$ & .46 \\
$\quad$ All RI & $17(13.7 \%)$ & $28(23.9 \%)$ & .04 \\
MI & & & \\
$\quad$ Q-wave MI & $5(4.0 \%)$ & $3(2.6 \%)$ & .52 \\
$\quad$ CK-MB elevation & $8(6.5 \%)$ & $10(8.6 \%)$ & .54 \\
$\quad$ All MI & $13(10.5 \%)$ & $13(11.1 \%)$ & .88 \\
Gastrointestinal & $2(1.6 \%)$ & $4(3.4 \%)$ & .37 \\
Limb ischemia & $2(1.6 \%)$ & $3(2.6 \%)$ & .60 \\
\hline
\end{tabular}

All data represent numbers and percentages of patients. TIA, Transient ischemic attach; $R I$, renal insufficiency; $M I$, myocardial infarction; $C K-M B$, creatine kinase isoenzyme MB. Adapted from Banbury MK, Kouchoukos NT, Allen KB, Slaughter MS, Weissman NJ, Berry GJ, et al; ICEM 2000 Investigators. Emboli capture using the Embol-X intraaortic filter in cardiac surgery: a multicentered randomized trial of 1,289 patients. Ann Thorac Surg. 2003;76:508-15; discussion 515. 
warranted." A larger randomized, controlled trial with patients at higher risk that incorporates imaging in addition to clinical endpoints is needed to determine the impact of filtration. Such a study is being sponsored by the National Heart, Lungs, and Blood Institute.

\section{Keith B. Allen, $M D^{a}$ \\ Keith Horvath, $M D^{b}$ \\ ${ }^{a}$ University of Missouri-Kansas City St Luke's Mid America Heart Institute Kansas City, Mo ${ }^{b}$ Cardiothoracic Surgery Research Program National Heart, Lungs, and Blood Institute National Institutes of Health Bethesda, Md}

\section{References}

1. Ye J, Webb JG. Embolic capture with updated intra-aortic filter during coronary artery bypass grafting and transaortic transcatheter aortic valve implantation: first-in-human experience. J Thorac Cardiovasc Surg. 2014;148:2905-10.

2. Banbury MK, Kouchoukos NT, Allen KB, Slaughter MS, Weissman NJ, Berry GJ, et al; ICEM 2000 Investigators. Emboli capture using the Embol-X intraaortic filter in cardiac surgery: a multicentered randomized trial of 1,289 patients. Ann Thorac Surg. 2003;76:508-15; discussion 515.

http://dx.doi.org/10.1016/j.jtcvs.2015.01.029

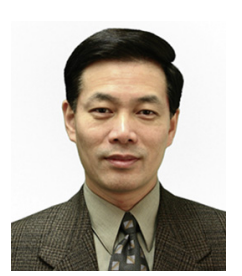

NEUROLOGIC IMPACT OF USING EMBOL-X INTRAAORTIC FILTER Reply to the Editor:

We greatly appreciate the comments of Allen and Horvath and entirely agree with their interpretation of the facts. We reported the safety and feasibility of using the new version of the EMBOL-X intraaortic filter during transaortic transcatheter aortic valve implantation and during conventional cardiac surgery, and we documented successful capture of embolic material. ${ }^{1}$ Although capturing emboli might be anticipated to decrease embolic events, it has been difficult to demonstrate a positive clinical effect. This may be in part because the majority of embolic cerebral events after transcatheter aortic valve implantation or conventional cardiac surgery are silent. We completely agree that a much larger, randomized study with a combination of imaging and clinical end points in patients who undergo either transaortic transcatheter aortic valve implantation or conventional cardiac surgery will be necessary for full evaluation of the potential for benefit or harm with any embolic control device.

Jian Ye, $M D$

John G. Webb, MD

St Paul's Hospital

University of British Columbia Vancouver, British Columbia, Canada

\section{Reference}

1. Ye J, Webb JG. Embolic capture with updated intra-aortic filter during coronary artery bypass grafting and transaortic transcatheter aortic valve implantation: first-in-human experience. J Thorac Cardiovasc Surg. 2014;148:2905-10.

http://dx.doi.org/10.1016/j.jtcvs.2015.02.011

\section{AORTIC SURGERY AND SPINAL COLLATERAL FLOW: A CALL FOR STRUCTURED APPROACHES TO FUNCTIONAL CHARACTERIZATION OF THE INTRASPINAL COLLATERAL SYSTEM \\ To the Editor:}

Generating knowledge of spinal collateral physiology spans over decades of scientific efforts. As will be demonstrated, historical and current knowledge of different specialized areas, such as orthopedic surgical, neurosurgical, neuroradiologic, and aortic surgical science, as well as basic research, need to be combined to generate a comprehensive picture of spinal collateral physiology.

Spinal collateral flow has been the subject of surgical large animal studies, which have proved to be the best available option among scientific models for spinal flow research. The mainstay experimental series from the Mount Sinai Hospital in New York ${ }^{1-3}$ stand out in this series of experimental efforts. After more than 20 years of translational and basic work shedding light on risks of central nervous deficits after thoracic aortic surgery, tendencies of resignation now can be observed. In their recent letter, Takayama and Borger ${ }^{4}$ come to the conclusion that spinal cord protection might be just as "simple" as keeping perfusion pressures high and to react on evident spinal ischemia as monitored and detected by evoked potentials. It is ignored that we are still far away from solid, evidence-based individual preoperative risk prediction of spinal ischemia. Strategies with predictive validity of spinal ischemia based on individual anatomic settings still need to be established.

Lazorthes and Gouaze, ${ }^{5,6}$ Lazorthes and colleagues, ${ }^{7}$ Lazorthes and Manelfe, ${ }^{8}$ Lazorthes and colleagues, ${ }^{9,10}$ Lazorthes and Zadeh, ${ }^{11}$ and Mehta ${ }^{12}$ are to be credited for probably the most important anatomic contributions to our understanding of the spinal collateral system, more specifically the intraspinal backup system. It seems as if their work somewhat faded into the background, which is not understandable especially regarding Lazorthes and colleagues' highly detailed and extensive investigations in cadavers. Their results have served as an anatomic basis for the numerous large animal studies by Griepp and colleagues at the Mount Sinai School of Medicine in New York City. Other early contributions are by Kadyi, ${ }^{13,14}$ Piscol, ${ }^{15}$ and Piscol and Remagen. ${ }^{16}$ Paradoxically, orthopedic researchers ${ }^{17-19}$ studying venous return from the spinal column primarily described an important piece of the intraspinal collateral system - the serial circular epidural 